\title{
How often does office hysteroscopy reveal pathology not previously noted on transvaginal ultrasound-a look at two typical reproductive endocrinology patient groups, including failed IVF-ET patients
}

\author{
B. Lawrenz • E. Neunhoeffer • S. Lessmann-Bechle • \\ D. Wallwiener $\cdot$ T. Fehm $\cdot$ S. Becker
}

Received: 18 November 2009 /Accepted: 5 March 2010/Published online: 31 March 2010

(C) Springer-Verlag 2010

\begin{abstract}
Transvaginal ultrasound and office hysteroscopy are established diagnostic tools for the evaluation of infertility patients, with hysteroscopy often considered as the more precise method to evaluate the uterine cavity and ultrasound as the more readily available and less invasive method. We look at both methods in two typical infertility patient groups, including one group with a history of failed in vitro fertilization-embryo transfer (IVF-ET) cycles. Eighty patients were prospectively evaluated. Forty patients presented with a history of failed embryo transfer despite repeat normal transvaginal ultrasound examinations. Forty infertility patients with abnormal ultrasound testing served as a group for comparison. All patients underwent office hysteroscopy. In the subgroup with abnormal ultrasound findings, office hysteroscopy confirmed the diagnosis in $75 \%$ of cases but did not add new information. In patients with a history of failed embryo transfer and at least twice normal ultrasound examinations, only $1 / 40$ patients $(2.5 \%)$ was noted to have a minor abnormality on office hysteroscopy not previously diagnosed on ultrasound. In infertility patients, including those with a history of failed IVF-ET cycles, repeat normal transvaginal ultrasound appears to be an efficient method for the determination of significant intrauterine abnormalities.
\end{abstract}

Declaration of interest The authors report no conflicts of interest. The authors alone are responsible for the content and writing of the paper.

B. Lawrenz $\cdot$ E. Neunhoeffer $\cdot$ S. Lessmann-Bechle

D. Wallwiener $\cdot$ T. Fehm $\cdot$ S. Becker $(\bowtie)$

Obstetrics and Gynecology, University Hospital Tübingen,

Tübingen, Germany

e-mail: sven.becker@med.uni-tuebingen.de
Keywords Transvaginal ultrasound - Office hysteroscopy Infertility patients $\cdot$ Failed IVF/ICSI cycles

\section{Background}

The exact causes of failed in vitro fertilization-embryo transfer (IVF-ET) cycles frequently remain unclear. While patient age and the quality of the transferred embryo play an important role, local factors like anatomic alterations of the uterine cavity can also be implicated. Intrauterine abnormalities like uterine septae, submucous myomas, polyps, or adhesions can prevent the successful implantation of a viable embryo [1-4].

Patients undergoing assisted reproductive interventions receive transvaginal ultrasound examinations at various stages of their treatment. Prior to therapy, the anatomic situation of the internal genitalia is assessed and repeatedly reviewed at the time of cycle monitoring. Other than the growth of the follicles, the uterine cavity and the structure and thickness of the endometrium are repeatedly evaluated.

Many reproductive endocrinologists use office hysteroscopy as a routine examination to further evaluate the intracavitary situation [3]. While office hysteroscopy is an established procedure with very low complication rates, it is slightly more invasive than transvaginal ultrasound, creating both additional costs and increased discomfort to the patient. The value of office hysteroscopy in the management of IVF patients, including IVF patients with failed cycles, has been well documented $[5,6]$. We were interested in the respective diagnostic value of transvaginal ultrasound when compared with office hysteroscopy in this context: Looking at a group of failed IVF-ET patients, we 
specifically evaluated the additional diagnostic value of office hysteroscopy when compared to repeat transvaginal ultrasound examinations. To further delineate the ability of office hysteroscopy to diagnose findings not previously noted on ultrasound, a comparison group with pathologic ultrasound findings was added to the study.

\section{Patients and methods}

A prospective analysis was performed over the course of 4 months, looking at two groups of patients. Group A consisted of IVF-ET patients with at least two failed assisted reproductive technology (ART) cycles who had never been diagnosed with any uterine abnormality during previous transvaginal ultrasound examinations performed prior to their infertility treatment nor during their ART cycles. During the study period, all patients presenting with this history were included in the study. As part of the routine monitoring of IVF-ET cycles, within the study group, at least three transvaginal ultrasound examinations were performed at a minimum. Ultrasound examinations were 2D/3D examinations performed by three experienced ultrasonographers (attending level, at least 3 years of professional experience: BL, SB, and EN). Group B consisted of patients with primary or secondary infertility or recurrent miscarriage without ongoing ART interventions, but with ultrasound examinations indicative of intrauterine abnormalities. Ultrasound findings considered "abnormal" were reviewed by two of the authors (BL, SB). All deviations from a normal-sized, regularly shaped uterus with a cavity appropriate for the day of the menstrual cycle were considered "pathological," including abnormal thickening of the endometrial stripe, calcifications, and myometrial protrusion into the cavity. To focus on intracavitary abnormalities, patients with myomas that were felt to be remote from the cavity (subserous, intramural) were excluded. The study protocol defined "abnormal hysteroscopy" as any hysteroscopy revealing the presence of endometrial or endocervical polyps, submucous myomas, or transmural myomas protruding and deforming the uterine cavity, intrauterine synechiae, intrauterine septae, or embryologic anatomic alterations of the uterus (arcuate uterus, etc.) as well as intrauterine adhesions.

After obtaining informed consent and according to the routine standard of care at our institution, all patients underwent office hysteroscopy without anesthesia. Additional analgesia was not necessary in the cases described. Hysteroscopy was performed during the first half of the menstrual cycle. Routine 4-mm hysteroscopes (Karl Storz Company) were used (Fig. 1). The distension medium was

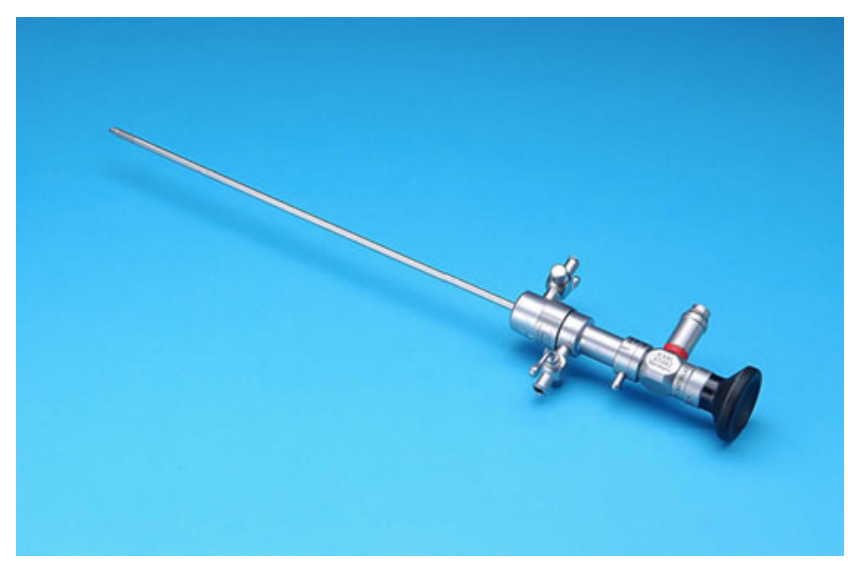

Fig. 1 Standard 4-mm hysteroscope for office hysteroscopy

sterile normal saline solution. No cervical dilatation was performed. All ultrasound examinations were performed by expert ultrasonographists as mentioned above, using a dedicated $6.5-\mathrm{MHz}$ transvaginal ultrasound probe. $2 \mathrm{D} / 3 \mathrm{D}$ imaging was performed. The study protocol did not differ from the routine workup procedures for IVF patients at our institution. All patients receive transvaginal ultrasound and office hysteroscopy. MRI examinations are reserved for specific questions (i.e., uterine embryologic abnormalities). Hysterosalpingographies (HSGs) are only rarely performed.

\section{Results/findings}

A total of 80 patients were enrolled in the study. In group A ( $\geq 2$ failed IVF-ET cycles), 40 patients were evaluated. Despite previously unremarkable transvaginal ultrasound examination, these patients underwent diagnostic outpatient hysteroscopy as described above prior to their subsequent infertility treatment.

Group B consisted of 40 patients who were treated because of infertility (primary or secondary) or recurrent miscarriage. These patients had had at least one transvaginal ultrasound indicative of abnormalities of or involving the uterine cavity. They underwent routine office hysteroscopy to further delineate the existing abnormality

Of 80 hysteroscopies, 79 were performed according to protocol, with adequate visualization of the uterine cavity. In one case (group B), severe cervical stenosis made an adequate procedure impossible. The patient was referred to routine diagnostic hysteroscopy under anesthesia. No complications such as perforation, infection, bleeding, or major patient discomfort occurred.

Patient age was between 27 and 41 years for group A (average age 34.7 years) and between 20 and 41 years for group B (average age 34.4 years). The most common indications for IVF-ET treatment were tubal sterility, longstanding idiopathic sterility, and male oligoasthenoterato- 
zoospermia (OAT syndrome) in both groups. Patient characteristics, including a comparison between both groups, are shown in Table 1.

Twenty-five patients in group A ( $\geq 2$ failed ART cycles) had initially presented with primary sterility and 15 with secondary sterility. Of the 15 patients with secondary sterility, eight patients had had one previous successful pregnancy. In seven patients, infertility treatment had led to one unsuccessful pregnancy with first trimester pregnancy loss.

The 40 patients in group A presented with a total of 145 previous IVF-ET cycles. The individual number of treatment cycles was between two (the minimum number for inclusion) and nine. Most patients in this group were referred to our center from other institutions.

\section{Results of hysteroscopy}

In group A, only one out of 40 patients $(2.5 \%)$ was found to have significant intrauterine findings on hysteroscopy that could have significantly decreased her chances of successful IVF-ET treatment. Despite previously unremarkable transvaginal ultrasound examinations, the patient was found to have a $0.5-\mathrm{cm}$ submucous/intramural myoma of the left sidewall (Fig. 2). The patient's history was remarkable for one previous pregnancy after ICSI that had ended in a first trimester loss, followed by three subsequently unsuccessful treatment cycles. The patient underwent operative hysterosopic myomectomy. In two patients, small intracervical polyps were noted. In both of these patients, the uterine cavity was unremarkable. In summary, our results show that in 39/40 ART patients with previously failed IVF treatments $(97.5 \%)$, the evaluation of the uterine cavity with repeat transvaginal ultrasound was correct and later confirmed by hysteroscopy. Only in one patient was a

Table 1 Patient characteristics

\begin{tabular}{lll}
\hline & Group A & Group B \\
\hline Number of patients & 40 & 40 \\
Range of ages & $27-41$ & $20-41$ \\
Average age & 34,7 & 34,37 \\
Previous ART cycles $(n)$ & $2-9$ & 0 \\
Primary sterility & $25(=62.5 \%)$ & $25(=62.5 \%)$ \\
Secondary sterility & $15(=37.5 \%)$ & $15(=37.5 \%)$ \\
Pathologic tubal factor & $9(=22.5 \%)$ & $6(=15.0 \%)$ \\
Idiopathic sterility & $6(=15.0 \%)$ & $18(=45 \%)$ \\
OAT syndrome & $25(=62.5 \%)$ & $6(=15.0 \%)$ \\
Recurrent miscarriage & 0 & $10(=25.0 \%)$ \\
\hline
\end{tabular}

${ }^{\mathrm{a}}$ Oligoasthenoteratozoospermia

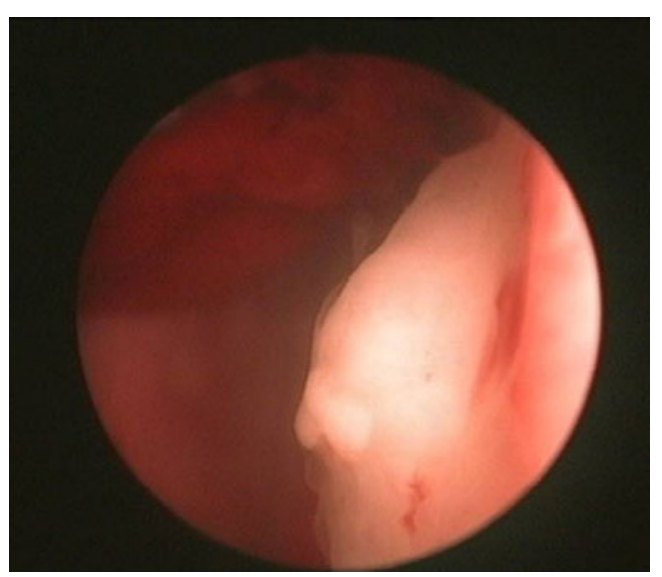

Fig. 2 Small submucous/intramural myoma arising from the left sidewall

potentially significant abnormality (submucous myoma) noted that had not been seen on ultrasound.

In group B, the ultrasound diagnosis leading to hysteroscopy was intracavitary polyp in 11 cases, myomas involving the cavity in 14 cases, and uterine septum in 12 cases. In three cases, abnormal appearance of the endometrium was the indication for hysteroscopy. Hysteroscopy subsequently confirmed the ultrasound diagnosis in 30/40 cases $(75 \%)$. In nine cases, hysteroscopy revealed a normal uterine cavity. Only in one patient $(2.5 \%)$ was the ultrasound diagnosis of endometrial polyp corrected: Hysteroscopy revealed the presence of a mostly intracavitary submucous myoma.

\section{Discussion}

Transvaginal ultrasound is an integral part of both diagnosis and treatment of reproductive endocrinology patients. In the hands of an expert ultrasonographer, it allows for exact evaluation of the internal genitalia in general and the uterus in particular. Specifically, it allows for an exact evaluation of the uterine cavity as well as the endometrium. Polyps, myomas, and uterine septae are readily seen and diagnosed. A normal, layered endometrial appearance at the time of ovulation has been shown to be associated with an increased pregnancy rate [7].

While ultrasound is an indirect, completely noninvasive diagnostic procedure, hysteroscopy allows for direct visualization of the uterine cavity and as such remains the ultimate diagnostic tool for the evaluation of intrauterine pathology. Even though it is a minimally invasive procedure, particularly in the technique generally referred to as "office hysteroscopy," i.e., hysteroscopy without anesthesia or cervical dilatations, it is more invasive than ultrasound. As a procedure, it adds additional costs and discomfort to the patient. 
Because of its ready availability as well as the generally low complication rate (though higher than transvaginal ultrasound), it has been suggested to use office hysteroscopy routinely, even in the absence of abnormal ultrasound findings. Looking at the background pathology, it is interesting to note that abnormalities of the uterine cavity are found with varying frequency in different studies, reflecting different definitions of what is abnormal. Table 2 summarizes the existing literature. While submucous myomas, obvious septae, and uterine synechia are generally defined as abnormal, small cervical polyps most likely will not have an appreciable effect on the pregnancy rate.

It is important to note that intracavitary polyps and myomas are usually easily diagnosed at transvaginal ultrasound examinations. Only uterine synechia are not readily seen on ultrasound. Because intrauterine abnormalities are a potentially treatable factor of infertility or sterility, various publications have looked at the question whether diagnostic hysteroscopy should be a standard procedure for every infertility patient [3, 8-11].

Clearly, documented intrauterine pathology has a significant negative effect on the pregnancy rate after IVF [12]. Some studies have found intrauterine pathology in a considerable number of infertility patients $[3,4]$. It is important to note that both Hinckley and Berger-Menz reported on a fairly nonspecific group of patients whereas Doldi et al. included endometrial hyperplasia and hypoplasia as intrauterine pathology.

However, the exact effect of documented intrauterine pathology, particularly discreet findings on ART success rates, has been questioned [8].

As a result, different recommendations exist. Some authors [10] recommend routine office hysteroscopy prior to the first cycle of stimulation while others limit the procedure to infertility patients with abnormal vaginal ultrasound findings. Shalev et al. [9] could demonstrate the high sensitivity and specificity of the transvaginal ultrasound approach with regard to the detection of intrauterine abnormalities. At a recent meeting of German IVF directors, only $3 \%$ of centers considered hysteroscopy prior to IVF mandatory [8]. Modern assisted reproductive interventions achieve pregnancy rates between $39 \%$ per embryo transfer in a 32 -year-old patient and $25 \%$ per embryo transfer in a 40-year-old patient (Deutsches IVF Register, Jahrbuch 2007). When the initial therapies fail, the question of whether transvaginal ultrasound is a sufficient tool to evaluate potential intrauterine pathology arises. Various studies have looked at this question [811]. Schiano et al. [13] used hysteroscopy in patients with two unsuccessful IVF cycles and found intrauterine pathology in $50 \%$ of patients. However, the study does not mention prior ultrasound results. Many of the pathologic findings were confined to the cervix (polyps, signs of via falsa after previous surgeries). After appropriate treatment, the pregnancy rate increased. Other studies have addressed the same question, looking at the value of office hysteroscopy after failed IVF cycles. Some studies have looked at the value of HSG when compared to office hysteroscopy. The value of hysteroscopy in this context has been well documented and is not being disputed $[5,6,11,14]$. We specifically compare the value of office hysteroscopy when preceded by repeat transvaginal ultrasonography, an approach less well studied in the literature, where mostly hysteroscopy and no intervention have been compared $[15,16]$. It has been criticized that even an expert ultrasonographer will not be able to see all intrauterine pathologies. Nawroth et al. [8] looked at this question and found intrauterine pathology in $10 \%$ of patients presenting with normal ultrasound. The majority of his findings, however, were intrauterine adhesions that vary considerably in terms of extent and clinical significance. In our patient group with unremarkable ultrasound findings, the incidence of adhesions is much lower.

In this study, we looked at 80 patients, half of them with a normal uterine ultrasound assessment, the other half with uterine pathology noted at ultrasound. Those patients with a normal ultrasound image of the uterine cavity were infertility patients with a history of minimum two previous failed ART attempts.

Table 2 Pathologic findings by hysteroscopy in infertility or sterility patients

\begin{tabular}{lcllllll}
\hline Publication & Patient number & $\begin{array}{l}\text { Normal } \\
\text { uterine cavity }\end{array}$ & Septum & $\begin{array}{l}\text { Endometrial } \\
\text { polyp }\end{array}$ & $\begin{array}{l}\text { Submucous } \\
\text { myoma }\end{array}$ & $\begin{array}{c}\text { Synechia } \\
\text { Abnormal uterine } \\
\text { cavity (total) }\end{array}$ \\
\hline Bordt et al. [2] & 26 & $88.5 \%$ & $\mathrm{~ns}$ & $3.8 \%$ & $7.6 \%$ & $\mathrm{~ns}$ & $11.5 \%$ \\
Hinckley et al. [3] & 1,000 & $62 \%$ & $0.5 \%$ & $32 \%$ & $3 \%$ & $3 \%$ & $38 \%$ \\
Berger-Menz et al. [4] & 235 & $52.7 \%$ & $\mathrm{~ns}$ & $18.7 \%$ & $3.4 \%$ & $3.4 \%$ & $1.5-25 \%$ \\
Nawroth et al. [8] & 375 & $\mathrm{~ns}$ & $2-3 \%$ & $\mathrm{~ns}$ & $\mathrm{~ns}$ & $10 \%$ \\
Shalev et al. [9] & 74 & $71.6 \%$ & $1.4 \%$ & $9.6 \%$ & $4.1 \%$ & $13.7 \%$ & $28.4 \%$ \\
Doldi et al. [11] & 300 & $60 \%$ & $\mathrm{~ns}$ & $26 \%$ & $\mathrm{~ns}$ & $\mathrm{~ns}$ \\
\hline
\end{tabular}

$n s$ not specified 
We find that repeated normal ultrasounds correctly predicts a normal cavity in almost $98 \%$ of cases, whereas even in abnormal ultrasound imaging, $22 \%$ of patients will have a normal cavity, underlining the high sensitivity of an unremarkable uterine cavity seen at sonography. We thus confirm the data presented by Shalev et al. [9] as mentioned above.

Despite looking at a selected clientele (failed IVF), these numbers differ from some of those quoted in the literature $[8,10,13,17]$. Possible explanations are the use of repeat ultrasound examination (at least three), including the option for 3D imaging in the hands of skilled and experienced physician ultrasonographers. Repeat ultrasound within this context appears to be an accurate and efficient method for the evaluation of the uterine cavity, comparable to the documented quality of office hysteroscopy.

\section{Conclusion}

Repeat transvaginal ultrasound examination performed by expert ultrasonographer in our series accurately diagnosed a normal uterine cavity in more than $97 \%$ of cases.

\section{References}

1. Rimbach S (2002) Invasive Diagnostik bei unerfülltem Kinderwunsch. Der Gynäkologe 35:1024-1037

2. Bordt J, Belkien L, Vancaillie T, Stening C, Schneider HPG (1984) Ergebnisse diagnostischer Hysteroskopien in einem IVF/ ET-Programm. Geburtshilfe Frauenheilkd 44:813-815

3. Hinckley MD, Milki AA (2003) 1,000 office hysteroscopies for infertility: feasibility and findings. Fertil Steril 80(Suppl 3):82-83

4. Berger-Menz E, Mueller MD, Eggimann T, Hänggi W, Dreher E (1999) Diagnostischer Wert der Hysteroskopie bei Sterilitäts- und Infertilitätsabklärungen. Geburtshilfe Frauenheilkd 59:413-415
5. Demirol A, Gurgan T (2004) Effect of treatment of intrauterine pathologies with offices hysteroscopy in patients with recurrent IVF failure. Reprod Biomed Online 8(5):590-594

6. Rama Raju GA, Shashi Kumari G, Krishna KM, Prakash GJ, Madan K (2006) Assessment of uterine cavity by hysteroscopy in assisted reproduction programme and its influence on pregnancy outcome. Arch Gynecol Obstet 274:160-164

7. Jarbela I, Sladkevicius P, Kelly S, Ojha K, Campbell S, Nargund G (2005) Evaluation of endometrial receptivity during in-vitro fertilization using three-dimensional power Doppler ultrasound. Ultrasound Obstet Gynecol 26:765-769

8. Nawroth F, Schmidt T, Foth D (2005) Zum Stellenwert der Hysteroskopie als Screeningmethode in der Sterilitätsdiagnostik. Geburtshilfe Frauenheilkd 65:139-143

9. Shalev J, Meizner I, Bar-Have I, Dicker D, Mashiach R, BenRafael Z (2000) Predictive value of transvaginal sonography performed before routine diagnostic hysteroscopy for evaluation of infertility. Fertil Steril 73:421-417

10. Feqhali J, Bakar J, Mavenga JM, Segard L, Hamou J, Driguez P, Belaisch-Allart J (2003) Systematic hysteroscopy prior to in vitro fertilization. Gynecol Obstet Fertil 31(2):127-131

11. Doldi N, Persico P, Di Sebastiano F, Marsiglio E, De Santis L, Rabellotti E, Fusi F, Brigante C, Ferari A (2005) Pathologic findings in hysteroscopy before in vitro fertilization-embryo transfer. Gynecol Endocrinol 21(4):235-237

12. Shamma F, Lee G, Gutmann J, Lavy G (1992) The role of office hysteroscopy in in vitro fertilization. Fertil Steril 58:1237-1239

13. Schiano A, Jourdain O, Papaxanthos A, Hocke C, Horovitz J, Dallay D (1999) The value of hysteroscopy after repeated implantation failures with in vitro fertilization. Contracept Fertil Sex 27(2):129-132

14. Mooney S, Milki A (2003) Effect of hysteroscopy performed in the cycle preceding controlled ovarian hyperstimulation on the outcome of in vitro fertilization. Fertil Steril 79(3):637-638

15. El-Toukhy T, Sunkara SK, Coomarasamy A, Grace J, Khalaf Y (2008) Outpatient hysteroscopy and subsequent IVF cycle outcome: a systematic review and meta-analysis. Reprod Biomed Online 16(5):712-719

16. Chung Y, Suh C, Choi Y, Kim J, Moon S, Kim S (2006) Effects of endometrial evaluation with office hysteroscopy in IVF-ET patients with repeated failures. Fertil Steril 86(Suppl 2):S152

17. Nawroth F, Foth D, Schmidt T (2003) Mini-hyperoscopy as an essential part of routine basic diagnostics in patients with primary infertility. J Am Assoc Gynecol Laparosc 10:396-398 